\title{
Stability and instability of individual nodes in multi-hop wireless CSMA/CA networks
}

\author{
Seva Shneer \\ Heriot-Watt University, Department of AMS \\ Edinburgh EH14 4AS, UK \\ v.shneer@hw.ac.uk
}

\author{
Peter M. van de Ven \\ Centrum Wiskunde en Informatica (CWI) \\ 1098 XG Amsterdam, Netherlands \\ ven@cwi.nl
}

\begin{abstract}
CSMA/CA is a popular random-access algorithm for wireless networks, but its stability properties are poorly understood. We consider a linear multi-hop network of three nodes where the neighbouring nodes interfere with each other and medium access is governed by the CSMA/CA algorithm. We assume that the source node is saturated and packets are forwarded through the network, each node transmitting towards its neighbour on the right. We demonstrate that the queue of the second node is saturated (unstable) and the queue of the third node is stable; this confirms heuristic arguments and simulation results found in the research literature. Providing a rigorous proof for the (in)stability of these nodes is complicated by the fact that neither queue is Markovian when considered in isolation, and the two queues are dependent. We then compute the limiting behavior of node 3 , and use this to determine the end-to-end throughput of the network. Finally, we vary the access probabilities of the nodes, and evaluate how this affects the stability and throughput of the system.
\end{abstract}

\section{Categories and Subject Descriptors}

G.3 [Probability and Statistics]: Queueing theory

\section{General Terms}

Performance, Theory

\section{Keywords}

Markov processes, random-access, stability, throughput

\section{INTRODUCTION}

Random-access protocols such as Carrier-Sense MultipleAccess with Collision Avoidance (CSMA/CA) 8 have gained much popularity for their ability to regulate the access of network nodes to a shared medium in a fully distributed fashion, and are for instance used in the IEEE 802.11 standard. A node using the CSMA/CA protocol attempts to access the medium after some random back-off time; nodes that sense activity of interfering nodes freeze their back-off counter until the medium is sensed idle.

The CSMA/CA algorithm has been widely studied in recent years, see, e.g., [3 $3,6,7$, and references therein. How- ever, only very little attention has been devoted to unsaturated CSMA/CA networks, that consider packets arriving according to some extraneous process rather than being always available. In 4,12 the authors consider a single-hop CMSA/CA network and show that it is very difficult to analyze any such network unless all nodes are mutually interfering. In 10 stability and throughput results are obtained for more general networks, but under the assumption that a node freezes its arrival process during a back-off period. The lack of results for even the smallest unsaturated CSMA/CA networks is representative of the difficulty of analyzing such models, caused by the fact that the packet-level dynamics affect the nodes' behavior in intricate ways.

We consider a linear CSMA/CA multi-hop network of three nodes, where node 1 is saturated and messages are forwarded to nodes 2 and 3, where they leave the network. Transmitting nodes will block their direct neighbor(s) from activating. The stability of nodes 2 and 3 and the end-toend throughput of this network has been studied in $2,5.11$, where the authors present heuristic arguments to assert that node 2 is unstable and node 3 is stable, and use this knowledge to compute the throughput.

Rigorous treatment of such networks is elusive because nodes 2 and 3 need to be considered in isolation in order to determine their (in)stability. However, in this case they are no longer Markovian, rendering inapplicable most of the machinery for proving stability. In this paper we analyze these non-Markovian processes, and demonstrate (in)stability. In fact, we generalize the access probability to the case that certain nodes are more aggressive, and study the stability behavior of the individual nodes and the resulting end-toend throughput.

The remainder of the paper is structured as follows. In Section 2 we present the model and in Section 3 we describe our main results. We conclude in Section 4 by highlighting various possible extensions and directions for future research.

\section{MODEL OUTLINE}

We consider a random-access network consisting of 3 nodes on a line, numbered 1,2,3. Time is slotted, and at the beginning of each time slot a feasible subset of nodes is activated for the duration of that slot. We denote the schedule of slot $t$ by $\mathbf{X}(t)=\left(X_{1}(t), X_{2}(t), X_{3}(t)\right) \in\{0,1\}^{3}$, with $X_{i}(t)=1$ if node $i$ is active in slot $t$ and $X_{i}(t)=0$ otherwise. The set of feasible activity states is then given as $\{(0,0,0),(1,0,0),(0,1,0),(0,0,1),(1,0,1)\}$. An active node will only activate if it has packets available, and if active it 
will transmit a single packet during a slot.

Node 1 is saturated, and packets are forwarded from node 1 to node 2 and from node 2 to node 3 . Once transmitted by node 3 , packets leave the system. Let $\mathbf{Q}=\left(Q_{2}(t), Q_{3}(t)\right)$ denote the queue length at time $t$, the evolution of which is given as

$$
\begin{aligned}
& Q_{2}(t+1)=Q_{2}(t)+X_{1}(t)-X_{2}(t), \\
& Q_{3}(t+1)=Q_{3}(t)+X_{2}(t)-X_{3}(t) .
\end{aligned}
$$

The node activity $\mathbf{X}(t)$ is independent between slots, and is generated as follows. Each slot is partitioned into a contention period and a transmission period. At the beginning of each contention period, if it has packets available, node $i$ draws a random back-off window $U_{i} \sim \operatorname{UNIF}\left(0, u_{i}\right)$ with some fixed $u_{i}>0, i=1,2,3$. Nodes activate when their back-off timer expires, unless a neighboring node is already transmitting. We introduce the following notation:

$$
\begin{aligned}
p_{23} & :=\mathbf{P}\left(\mathbf{X}(t)=(0,1,0) \mid Q_{2}(t), Q_{3}(t) \geq 1\right) \\
& =\mathbf{P}\left(U_{2}(t)<\min \left\{U_{1}(t), U_{3}(t)\right\}\right), \\
p_{2} & :=\mathbf{P}\left(\mathbf{X}(t)=(0,1,0) \mid Q_{2}(t) \geq 1, Q_{3}(t)=0\right) \\
& =\mathbf{P}\left(U_{2}(t)<U_{1}(t)\right) .
\end{aligned}
$$

Because of the saturation assumption and the nature of the access mechanism, node activity will only take values in $\mathbf{X}(t) \in\{(1,0,0),(0,1,0),(1,0,1)\}$. Note that, if $Q_{2}(t)=0$, $\mathbf{X}=\left(1,0, \mathbb{1}_{\left\{Q_{3}(t)>1\right\}}\right)$, so $p_{23}$ and $p_{2}$ completely characterize the distribution of the node activity, and thus the evolution of $\mathbf{Q}(t)$. Moreover, $p_{23}$ and $p_{2}$ can each achieve any value in $(0,1)$ by choosing appropriate $u_{i}, i=1,2,3$. In the remainder of this paper we shall take the $p_{23}, p_{2}$ as a starting point of our analysis, without specifying the parameters of the CSMA/CA algorithm that achieves these probabilities. In view of (1) and (2), we assume $p_{23}<p_{2}$ throughout.

By evaluating the dynamics, it is readily seen that the function $Q_{2}(t)+Q_{3}(t)$, representing the total number of messages at nodes 2 and 3 , does not decrease with probability 1. Thus, the Markov process $\mathbf{Q}$ is transient for all $p_{2}, p_{23}$, which precludes a stationary analysis of the system. However, we shall see in Section 3 that by evaluating the stability of each node individually, we are able to compute the end-to-end throughput of the network despite the transience of the underlying Markov chain.

\section{MAIN RESULTS}

Depending on the choice of $p_{23}$, the network exhibits different behavior. In this note we limit ourselves to the case $p_{23}<1 / 2$. First we can show that node 3 is stable, irrespective of $p_{2}$.

Proposition 1. Let $p_{23}<1 / 2$, then

$$
\liminf _{t \rightarrow \infty} Q_{3}(t)<\infty \quad \text { a.s }
$$

Proof. Denote $q_{2}:=Q_{2}(t), q_{3}:=Q_{3}(t)$ and let $q_{3} \geq 1$. It is immediate that

$$
\mathbf{E}\left(Q_{3}(t+1) \mid \mathcal{H}_{\mathbf{Q}_{\mathbf{3}}}(t)\right)= \begin{cases}q_{3}-1, & \text { if } q_{2}=0, \\ q_{3}-\left(1-2 p_{23}\right), & \text { otherwise, }\end{cases}
$$

where $\mathcal{H}_{\mathbf{Q}_{\mathbf{3}}}(t)$ denotes the history of the process $\left\{Q_{3}(u)\right\}_{u \geq 0}$, up to and including time $t$. Hence, irrespective of $q_{2}$,

$$
\mathbf{E}\left(Q_{3}(t+1) \mid \mathcal{H}_{\mathbf{Q}_{\mathbf{3}}}(t)\right) \leq q_{3}
$$

as long as $q_{3} \geq 1$. We can now use Theorem 2.1 from 9 to conclude the proof.

Next, we can show that node 2 is not stable whenever $p_{2}<p_{2}^{*}:=\frac{1}{2}\left(2+p_{23}-\sqrt{p_{23}\left(4+p_{23}\right)}\right)$.

Lemma 1. Let $p_{23}<1 / 2$ and $p_{2}<p_{2}^{*}$, then $Q_{2}(t) \rightarrow \infty$ a.s. as $t \rightarrow \infty$.

Proof. Note that it is sufficient to show that $Q_{2}(2 t) \rightarrow \infty$ a.s. as $t \rightarrow \infty$. Indeed, as node 2 can at most transmit a single message in a time slot, $Q_{2}(2 t+1) \geq Q_{2}(2 t)-1$, and the result follows.

Fix a value $Q_{2}(2 t)=q_{2} \geq 3$, and observe that $Q_{2}(2(t+$ 1)) $\in\left\{q_{2}-2, q_{2}, q_{2}+2\right\}$. Denote $Q_{2}(t)=q_{2}, Q_{3}(t)=q_{3}$, and

$$
\begin{aligned}
p_{+2}\left(q_{2}, q_{3}\right) & :=\mathbf{P}\left(Q_{2}(2(t+1))=q_{2}+2\right), \\
p_{0}\left(q_{2}, q_{3}\right) & :=\mathbf{P}\left(Q_{2}(2(t+1))=q_{2}\right), \\
p_{-2}\left(q_{2}, q_{3}\right) & :=\mathbf{P}\left(Q_{2}(2(t+1))=q_{2}-2\right) .
\end{aligned}
$$

Observe that, irrespective of $q_{3}, p_{+2}\left(q_{2}, q_{3}\right) \geq\left(1-p_{2}\right)^{2}$ and $p_{-2}\left(q_{2}, q_{3}\right) \leq p_{2} p_{23}$. Since $p_{23}<p_{2}<p_{2}^{*}$, it is readily verified that $p_{+2}\left(q_{2}, q_{3}\right)>p_{-2}\left(q_{2}, q_{3}\right)$ for any values of $q_{3}$.

The rest of the proof will use the ideas of the proof of transience in Theorem 3.1 of 9 . Introduce a process

$$
Y(t)=1-\frac{1}{Q_{2}(2 t)+1}
$$

and remark that $0 \leq Y(t) \leq 1$ a.s. for all $t$. It is clearly sufficient to show that $Y(t) \rightarrow 1$ a.s. as $t \rightarrow \infty$. For this, according to Theorem 2.2 from 9 , it is sufficient to show that

$$
\mathbf{E}\left(Y(t+1) \mid Y(t)=y, \mathcal{H}_{Y}(t)\right) \geq y
$$

for large enough values of $y$, where $\mathcal{H}_{Y}(t)$ is the history of $Y(u)$ up to time $t$.

Now denote $y=Y(t)$ and $q_{2}(y)=y /(1-y)$, and let $Q_{2}(t)=q_{2}(y)$. Observe that $q_{2}(y)$ is increasing in $y$. By rewriting (3), we obtain

$$
\begin{aligned}
& 1-p_{+2}\left(q_{2}(y), q_{3}\right) \frac{1}{q_{2}(y)+3}-p_{0}\left(q_{2}(y), q_{3}\right) \frac{1}{q_{2}(y)+1} \\
& -p_{-2}\left(q_{2}(y), q_{3}\right) \frac{1}{q_{2}(y)-1} \geq 1-\frac{1}{q_{2}(y)+1}
\end{aligned}
$$

for large enough $q_{2}(y)$. After some calculations this can be shown to simplify to

$$
\frac{p_{-2}\left(q_{2}(y), q_{3}\right)}{q_{2}(y)-1} \leq \frac{p_{+2}\left(q_{2}(y), q_{3}\right)}{q_{2}(y)+3} .
$$

This indeed holds for large enough $q_{2}(y)$ as $p_{+2}\left(q_{2}(y), q_{3}\right)>$ $p_{-2}\left(q_{2}(y), q_{3}\right)$.

We have demonstrated that for $p_{23}<1 / 2$ and $p_{2}<p_{2}^{*}$, $Q_{2}(t)$ is not stable, but $Q_{3}(t)$ is. A natural question to ask now is what is the limiting behavior of $Q_{3}(t)$, and how can this be used to determine the end-to-end throughput of the network. Since $Q_{3}(t)$ in isolation is not a Markov process, determining its limiting behavior is not straightforward, and we first require the following auxiliary result.

Specifically, let us introduce a Markov chain $\widetilde{Q}_{3}(t)$ with the following transition probabilities:

$$
\begin{aligned}
& \mathbf{P}\left(\widetilde{Q}_{3}(t+1)=j \mid \widetilde{Q}_{3}(t)=i\right) \\
= & \mathbf{P}\left(Q_{3}(t+1)=j \mid Q_{3}(t)=i, Q_{2}(t)>0\right)
\end{aligned}
$$


and define $p_{i j}^{t}:=\mathbf{P}\left(\widetilde{Q}_{3}(t)=j \mid \widetilde{Q}_{3}(0)=i\right)$.

The Markov chain $\widetilde{Q}_{3}(t)$ behaves as $Q_{3}(t)$ in case $Q_{2}(t)$ always has packets available, and we intend to show that the limiting behavior of these two processes is indeed the same. Although this is intuitively clear since we show in Lemma 1 that $Q_{2}(t)$ is unstable, it takes some effort to show this.

The process $\widetilde{Q}_{3}(t)$ is a one-dimensional birth-death process with negative drift, and its limiting distribution can be readily obtained from the balance equations. The following result is thus stated without proof.

Lemma 2. Let $p_{23}<1 / 2$, then $\widetilde{Q}_{3}(t)$ has a limiting distribution $\left(\pi_{j}\right)_{j=1,2, . .}$, i.e., $p_{i j}^{t} \rightarrow \pi_{j}$ as $t \rightarrow \infty$, regardless of $i$. It is given as

$$
\pi_{j}= \begin{cases}\frac{1-2 p_{23}}{1-2 p_{23}+p_{2}}, & \text { if } j=0, \\ \pi_{0} \frac{p_{2} p_{23}^{j}}{\left(1-p_{23}\right)^{j}}, & \text { if } j \geq 1 .\end{cases}
$$

We are now in position to state and prove the main result on the limiting behavior of $Q_{3}(t)$ and the end-to-end throughput of the network.

TheOREM 1. Let $p_{23}<1 / 2$ and $p_{2}<p_{2}^{*}$, then

$$
\mathbf{P}\left(Q_{3}(t)=j \mid Q_{3}(0)=i\right) \rightarrow \pi_{j} \quad \text { as } t \rightarrow \infty,
$$

regardless of $i$, with $\left(\pi_{j}\right)_{j=0,1, \ldots}$ from (4).

Proof. This is a direct application of Lemma 1 from 1$]$ and Lemmas 1 and 2

The end-to-end throughput $\theta$ of the network is simply the rate at which packets leave the third node, i.e.

$$
\theta\left(p_{23}, p_{2}\right):=\left(1-\pi_{0}\right)\left(1-p_{23}\right)=\frac{\left(1-p_{23}\right) p_{2}}{1-2 p_{23}+p_{2}} .
$$

It is worth noting that Theorem 1 covers the case considered in 2, 5.11 in which all nodes have equal probability of activating first. In this case, $p_{23}=1 / 3$ and $p_{2}=1 / 2$, and it follows from Theorem 1 that node 2 is unstable, node 3 is stable, and the end-to-end throughput equals $\theta(1 / 3,1 / 2)=$ $2 / 5$.

We conjecture that $p_{2}<p_{2}^{*}$ is only a technical condition facilitating our proof and that instability of node 2 holds for $p_{2} \geq p_{2}^{*}$ as well. Should this indeed be the case, one can see from (5) that the throughput is an increasing function of both $p_{2}$ and $p_{23}$ and by making $p_{23}$ close to $1 / 2$ and $p_{2}$ close to 1 , we can make the throughput of the system close to $1 / 2$ (which is of course the highest possible throughput given the system constraints).

\section{OUTLOOK}

The results presented in this paper suggest several natural extensions. For instance, we can extend Proposition 1 in a straightforward manner to networks of arbitrary length and interference range (distance within which nodes are blocked from activating simultaneously). It is not immediately clear how the instability result of Lemma 1 can be extended to larger networks, although the heuristics presented in 11 suggest that this should indeed hold. In order to facilitate a rigorous analysis of larger networks, one needs to extend Lemma 1 from 11 to higher-dimensional systems, which constitutes an interesting direction for future research.

For the case $p_{23}>1 / 2$, we can prove the following analogue of Proposition 1 for the buffer of node 2 (with similar proof).
Proposition 2. If $p_{23}>1 / 2$, then

$$
\liminf _{t \rightarrow \infty} Q_{2}(t)<\infty \quad \text { a.s.. }
$$

The behaviour of the third note in case $p_{23}>1 / 2$ is more involved and does not appear to allow for straightforward analysis. Observe that $Q_{2}(t)+Q_{3}(t)$ will diverge a.s., and that this quantity can only increase whenever $Q_{3}(t)=0$. This seems to suggest that $Q_{3}(t)$ will hit 0 infinitely often and is thus in some sense stable. On the other hand, we know that the total number of packets in nodes 2 and 3 diverges and the system thus seems to exhibit a rather unusual behavior. Moreover, both nodes 2 and 3 seem to be in some sense stable and they also depend on each other - to the best of our knowledge, there are no theoretical results on such processes and this suggests an interesting avenue for future research.

\section{REFERENCES}

[1] I. Adan and G. Weiss. A skill based parallel service system under FCFS-ALIS-steady state, overloads, and abandonments. Stochastic Systems, 4(1):250-299, 2014.

[2] A. Aziz, S. Shneer, and P. Thiran. Wireless multi-hop networks beyond capacity. In 19th IEEE International Workshop on Local and Metropolitan Area Networks (LANMAN), Brussels, Belgium, April 10-12 2013.

[3] R. Boorstyn and A. Kershenbaum. Throughput analysis of multihop packet radio. In Proc. ICC, pages 1361-1366, 1980.

[4] F. Cecchi, S. Borst, and J. Van Leeuwaarden. Throughput of CSMA networks with buffer dynamics. Performance Evaluation, 79:216-234, 2014.

[5] D. Denteneer, S. Borst, P. van de Ven, and G. Hiertz. IEEE 802.11 s and the philosophers' problem. Statistica Neerlandica, 62(3):283-298, 2008.

[6] M. Durvy and P. Thiran. A packing approach to compare slotted and non-slotted medium access control. In Proc. Infocom, Barcelona, Spain, April 23-29 2006.

[7] M. Garetto, T. Salonidis, and E. Knightly. Modeling per-flow throughput and capturing starvation in CSMA multi-hop wireless networks. IEEE/ACM Trans. Netw., 16(4):864-877, 2008.

[8] L. Kleinrock and F. Tobagi. Packet switching in radio channels: part I - carrier sense multiple-access modes and their throughput-delay characteristics. IEEE Trans. Commun., 23(12):1400-1416, 1975.

[9] J. Lamperti. Criteria for the recurrence or transience of stochastic process. i. J. Math. Anal. Appl., $1(3): 314-330,1960$.

[10] R. Laufer and L. Kleinrock. The capacity of wireless CSMA/CA networks. IEEE Trans. Netw. (to appear), 2015.

[11] V. Shneer and P. van de Ven. Comparing slotted and continuous CSMA: throughputs and fairness. In Proc. Performance, pages 35-37, Amsterdam, NL, October 18-20 2011.

[12] P. van de Ven, S. Borst, J. van Leeuwaarden, and A. Proutière. Insensitivity and stability of random-access networks. Performance Evaluation, 67(11):1230-1242, 2010. 